\title{
A Truthful Mechanism for Offline Ad Slot Scheduling
}

\author{
Jon Feldman ${ }^{1}$, S. Muthukrishnan ${ }^{1}$, Evdokia Nikolova $^{2}$, and Martin Pál ${ }^{1}$ \\ 1 Google, Inc. Email: \{jonfeld,muthu,mpal\}@google.com \\ 2 Massachusetts Institute of Technology ${ }^{\star}$. Email: nikolova@mit.edu
}

\begin{abstract}
We consider the Offline Ad Slot Scheduling problem, where advertisers must be scheduled to sponsored search slots during a given period of time. Advertisers specify a budget constraint, as well as a maximum cost per click, and may not be assigned to more than one slot for a particular search.

We give a truthful mechanism under the utility model where bidders try to maximize their clicks, subject to their personal constraints. In addition, we show that the revenue-maximizing mechanism is not truthful, but has a Nash equilibrium whose outcome is identical to our mechanism. As far as we can tell, this is the first treatment of sponsored search that directly incorporates both multiple slots and budget constraints into an analysis of incentives.

Our mechanism employs a descending-price auction that maintains a solution to a certain machine scheduling problem whose job lengths depend on the price, and hence is variable over the auction. The price stops when the set of bidders that can afford that price pack exactly into a block of ad slots, at which point the mechanism allocates that block and continues on the remaining slots. To prove our result on the equilibrium of the revenue-maximizing mechanism, we first show that a greedy algorithm suffices to solve the revenue-maximizing linear program; we then use this insight to prove that bidders allocated in the same block of our mechanism have no incentive to deviate from bidding the fixed price of that block.
\end{abstract}

\footnotetext{
* This work was done while the author was visiting Google, Inc., New York, NY.
} 


\section{Introduction}

Sponsored search is an increasingly important advertising medium, attracting a wide variety of advertisers, large and small. When a user sends a query to a search engine, the advertisements are placed into slots, usually arranged linearly down the page. These slots have a varying degree of exposure, often measured in terms of the probability that the ad will be clicked; a common model is that the higher ads tend to attract more clicks. The problem of allocating these slots to bidders has been addressed in various ways. The most common method is to allocate ads to each search independently via a generalized second price (GSP) auction, where the ads are ranked by (some function of) their bid, and placed into the slots in rank order. (See [18] for a survey of this area.)

There are several important aspects of sponsored search not captured by the original models. Most advertisers are interested in getting many clicks throughout the day on a variety of searches, not just a specific slot on a particular search query. Also, many advertisers have budget constraints, where they do not allow the search engine to spend more than their budget during the day. Finally, search engines may have some knowledge about the distribution of queries that will occur during the day, and so should be able to make more efficient allocation decisions than just simple ranking.

The Offline Ad Slot Scheduling problem is this: given a set of bidders with bids (per click) and budgets (per day), and a set of slots over the entire day where we know the expected number of clicks in each slot, find a schedule that places bidders into slots. The schedule must not place a bidder into two different slots at the same time. In addition, we must find a price for each bidder that does not exceed the bidder's budget constraint, nor their per-click bid. (See Section 1.3 for a formal statement of the problem.)

A good algorithm for this problem will have high revenue. Also, we would like the algorithm to be truthful; i.e., each bidder will be incented to report her true bid and budget. In order to prove something like this, we need a utility function for the bidder that captures the degree to which she is happy with her allocation. Natural models in this context (with clicks, bids and budgets) are click-maximization - where she wishes to maximize her number of clicks subject to her personal bid and budget constraints, or profit-maximization - where she wishes to maximize her profit (clicks $\times$ profit per click). In this paper we focus on click-maximization. ${ }^{3}$

We present an efficient mechanism for Offline Ad Slot Scheduling and prove that it is truthful. We also prove that the revenue-optimal mechanism for Offline

\footnotetext{
${ }^{3}$ Our choice is in part motivated by the presence of budgets, which have a natural interpretation in this application: if an overall advertising campaign allocates a fixed portion of its budget to online media, then the agent responsible for that budget is incented to spend the entire budget to maximize exposure. In contrast, under the profit-maximizing utility, a weak motivation for budgets is a limit on liquidity. Also, our choice of utility function is out of analytical necessity: Borgs et al. [4] show that under some reasonable assumptions, truthful mechanisms are impossible under a profit-maximizing utility.
} 
Ad Slot Scheduling is not truthful, but has a Nash equilibrium (under the same utility model) whose outcome is equivalent to our mechanism; this result is strong evidence that our mechanism has desirable revenue properties. Our results generalize to a model where each bidder has a personal click-through-rate that multiplies her click probability.

As far as we can tell, this is the first treatment of sponsored search that directly incorporates both multiple positions and budget constraints into an analysis of incentives (see Section 1.2 for a survey of related work). In its full generality, the problem of sponsored search is more complex than our model; e.g., since the query distribution is noisy, good allocation strategies need to be online and adaptive. Also, our mechanism is designed for a single query type, whereas advertisers are interested in enforcing their budget across multiple query types. However, the tools used in this paper may be valuable for deriving more general mechanisms in the future.

1.1 Methods and Results. A natural mechanism for Offline Ad Slot Scheduling is the following: find a feasible schedule and a set of prices that maximizes revenue, subject to the bidders' constraints. It is straightforward to derive a linear program for this optimization problem, but unfortunately this is not a truthful mechanism (see Example 1 in Section 2). However, there is a direct truthful mechanism - the price-setting mechanism we present in this paperthat results in the same outcome as an equilibrium of the revenue-maximizing mechanism.

We derive this mechanism (and prove that it is truthful) by starting with the single-slot case in Section 2, where two extreme cases have natural, instructive interpretations. With only bids (and unlimited budgets), a winner-take-all mechanism works; with only budgets (and unlimited bids) the clicks are simply divided up in proportion to budgets. Combining these ideas in the right way results in a natural descending-price mechanism, where the price (per click) stops at the point where the bidders who can afford that price have enough budget to purchase all of the clicks.

Generalizing to multiple slots requires understanding the structure of feasible schedules, even in the special budgets-only case. In Section 3 we solve the budgets-only case by characterizing the allowable schedules in terms of the solution to a classical machine scheduling problem (to be precise, the problem $Q|p m t n| C_{\max }$ [13]). The difficulty that arises is that the lengths of the jobs in the scheduling problem actually depend on the price charged. Thus, we incorporate the scheduling algorithm into a descending-price mechanism, where the price stops at the point where the scheduling constraints are tight; at this point a block of slots is allocated at a fixed uniform price (dividing the clicks equally by budget) and the mechanism iterates. We present the full mechanism in Section 4 by incorporating bids analogously to the single-slot case: the price descends until the set of bidders that can afford that price has enough budget to make the scheduling constraints tight. A tricky case arises when a new bidder appears whose budget violates the scheduling constraints; in this case the budget of this "threshold" bidder is reduced to make them tight again. Finally in 
Section 4.2 we show that the revenue-optimal mechanism has a Nash equilibrium whose outcome is identical to our mechanism. This follows from the fact that if all the bidders in a block declare a bid (roughly) equal to the price of the block, nobody has an incentive to deviate, since every bidder is charged exactly her bid, and the clicks are divided up equally by budget.

1.2 Related Work. There are some papers on sponsored search that analyze the generalized second-price (GSP) auction, which is the auction currently in use at Google and Yahoo. The equilibria of this auction are characterized and compared with VCG $[9,17,2,22]$. Here the utility function is the profit-maximizing utility where each bidder attempts to maximize her clicks $\times$ profit per click, and budget constraints are generally not treated.

Borgs et al. [4] consider the problem of budget-constrained bidders for multiple items of a single type, with a utility function that is profit-maximizing, modulo being under the budget (being over the budget gives an unbounded negative utility). They give a truthful mechanism allocating some portion of the items that is revenue-optimal, and prove that in their model, under reasonable assumptions, truthful mechanisms that allocate all the units are impossible. Our work is different both because of the different utility function and the generalization to multiple slots with a scheduling constraint. Using related methods, Mahdian et al. [19] consider an online setting where an unknown number of copies of an item arrive online, and give a truthful mechanism with a constant competitive ratio guarantee.

There is some work on algorithms for allocating bidders with budgets to keywords that arrive online, where the bidders place (possibly different) bids on particular keywords $[20,19]$. The application of this work is similar to ours, but their concern is purely online optimization; they do not consider the gametheoretic aspects of the allocation. Abrams et al. [1] derive a linear program for the offline optimization problem of allocating bidders to queries, and handle multiple positions by using variables for "slates" of bidders. Their LP is related to ours, but again they do not consider game-theoretic aspects of their proposed allocations.

Bidder strategies for keyword auctions in the presence of budget constraints have also been considered $[11,21,6,5]$. Generally these papers are not concerned with mechanism design, but there could be some interesting relationships between the models in these papers and the one we study here.

In our setting one is tempted to apply a Fisher Market model: here $m$ divisible goods are available to $n$ buyers with money $B_{i}$, and $u_{i j}(x)$ denotes $i$ 's utility of receiving $x$ amount of good $j$. It is known $[3,10,7]$ that under certain conditions a vector of prices for goods exists such that the market clears, in that there is no surplus of goods, and all the money is spent. Furthermore, this price vector can be found efficiently [8]. The natural way to apply a Fisher model to a slot auction is to regard the slots as commodities and have the utilities be in proportion to the number of clicks. However this becomes problematic because there does not seem to be a way to encode the scheduling constraints in the Fisher model; this 
constraint could make an apparently "market-clearing" equilibrium infeasible, and indeed plays a central role in our investigations.

1.3 Our Setting. We define the Offline Ad Slot Scheduling problem as follows. We have $n>1$ bidders interested in clicks. Each bidder $i$ has a budget $B_{i}$ and a maximum cost-per-click (max-cpc) $m_{i}$. Given a number of clicks $c_{i}$, and a price per click $p$, the utility $u_{i}$ of bidder $i$ is $c_{i}$ if both the true max-cpc and the true budget are satisfied, and $-\infty$ otherwise. In other words, $u_{i}=c_{i}$ if $p \leq m_{i}$ and $c_{i} p \leq B_{i}$; and $u_{i}=-\infty$ otherwise. We have $n^{\prime}$ advertising slots where slot $i$ receives $D_{i}$ clicks during the time interval $[0,1]$. We assume $D_{1}>D_{2}>\ldots>D_{n^{\prime}}$.

In a schedule, each bidder is assigned to a set of (slot, time interval) pairs $(j,[\alpha, \beta))$, where $j \leq n^{\prime}$ and $0 \leq \alpha<\beta \leq 1$. A feasible schedule is one where no more than one bidder is assigned to a slot at any given time, and no bidder is assigned to more than one slot at any given time. (Formally, the intervals for a particular slot do not overlap, and the intervals for a particular bidder do not overlap.) A feasible schedule can be applied as follows: when a user query comes at some time $\alpha \in[0,1]$, the schedule for that time instant is used to populate the ad slots. If we assume that clicks come at a constant rate throughout the interval $[0,1]$, the number of clicks a bidder is expected to receive from a schedule is the sum of $(\beta-\alpha) D_{j}$ over all pairs $(j,[\alpha, \beta))$ in its schedule. ${ }^{4}$

A mechanism for Offline Ad Slot Scheduling takes as input a declared budget $B_{i}$ and declared max-cpc (the "bid") $b_{i}$, and returns a feasible schedule, as well as a price per click $p_{i} \leq b_{i}$ for each bidder. The schedule gives some number $c_{i}$ of clicks to each bidder $i$ that must respect the budget at the given price; i.e., we have $p_{i} c_{i} \leq B_{i}$.

The revenue of a mechanism is $\sum_{i} p_{i} c_{i}$. We say a mechanism is truthful if it is a weakly dominant strategy to declare one's true budget and max-cpc; i.e., for any particular bidder $i$, given any set of bids and budgets declared by the other bidders, declaring her true budget $B_{i}$ and max-cpc $m_{i}$ maximizes her utility $u_{i}$. A (pure strategy) Nash equilibrium is a set of declared bids and budgets such that no bidder wants to change her declaration of bid or budget, given that all other declarations stay fixed. An $\epsilon$-Nash equilibrium is a set of bids and budgets where no bidder can increase her utility by more than $\epsilon$ by changing her bid or budget.

Throughout the paper we assume some arbitrary lexicographic ordering on the bidders, that does not necessarily match the subscripts. When we compare two bids $b_{i}$ and $b_{i^{\prime}}$ we say that $b_{i} \succ b_{i^{\prime}}$ iff either $b_{i}>b_{i^{\prime}}$, or $b_{i}=b_{i^{\prime}}$ but $i$ occurs first lexicographically.

\section{One Slot Case}

In this section we consider the case $k=1$, where there is only one advertising slot, with some number $D:=D_{1}$ of clicks. We will derive a truthful mechanism

\footnotetext{
${ }^{4}$ All our results generalize to the setting where each bidder $i$ has a "click-through rate" $\gamma_{i}$ and receives $(\beta-\alpha) \gamma_{i} D_{j}$ clicks (see Section 5). We leave this out for clarity.
} 
for this case by first considering the two extreme cases of infinite bids and infinite budgets. The proofs of the theorems in this section are in Appendix A.

Suppose all budgets $B_{i}=\infty$. Then, our input amounts to bids $b_{1} \succ b_{2} \succ$ $\ldots \succ b_{n}$. Our mechanism is simply to give all the clicks to the highest bidder. We charge bidder 1 her full price $p_{1}=b_{1}$. We claim that reporting the truth is a weakly dominant strategy for this mechanism. Clearly all bidders will report $b_{i} \leq m_{i}$, since the price is set to $b_{i}$ if they win. The losing bidders cannot gain from decreasing $b_{i}$. The winning bidder can lower her price by lowering $b_{i}$, but this will not gain her any more clicks, since she is already getting all $D$ of them.

Now suppose all bids $b_{i}=\infty$. In this case, our input is just a set of budgets $B_{1}, \ldots, B_{n}$, and we need to allocate $D$ clicks, with no ceiling on the per-click price. Here we apply a simple rule related to pricing schemes for network bandwidth (see $[16,15])$ : Let $\mathcal{B}=\sum_{i} B_{i}$. Now to each bidder $i$, allocate $\left(B_{i} / \mathcal{B}\right) D$ clicks. Set all prices the same: $p_{i}=p=\mathcal{B} / D$. The mechanism guarantees that each bidder exactly spends her budget, thus no bidder will report $B_{i}^{\prime}>B_{i}$. Now suppose some bidder reports $B_{i}^{\prime}=B_{i}-\Delta$, for $\Delta>0$. Then this bidder is allocated $D\left(B_{i}-\Delta\right) /(\mathcal{B}-\Delta)$ clicks, which is less than $D\left(B_{i} / \mathcal{B}\right)$, since $n>1$ and all $B_{i}>0$.

2.1 Greedy First-Price Mechanism. A natural mechanism for the general single-slot case is to solve the associated "fractional knapsack" problem, and charge bidders their bid; i.e., starting with the highest bidder, greedily add bidders to the allocation, charging them their bid, until all the clicks are allocated. We refer to this as the greedy first-price (GFP) mechanism. Though natural (and revenue-maximizing as a function of bids) this mechanism is easily seen to be not truthful:

Example 1. Suppose there are two bidders and $D=120$ clicks. Bidder 1 has $\left(m_{1}=\$ 2\right.$, $\left.B_{1}=\$ 100\right)$ and bidder 2 has $\left(m_{2}=\$ 1, B_{2}=\$ 50\right)$. In the GFP mechanism, if both bidders tell the truth, then bidder 1 gets 50 clicks for $\$ 2$ each, and 50 of the remaining 70 clicks go to bidder 2 for $\$ 1$ each. However, if bidder 1 instead declares $b_{1}=\$ 1+\epsilon$, then she gets (roughly) 100 clicks, and bidder 2 is left with (roughly) 20 clicks.

The problem here is that the high bidders can get away with bidding lower, thus getting a lower price. The difference between this and the unlimited-budget case above is that a lower price now results in more clicks. It turns out that in equilibrium, this mechanism will result in an allocation where a prefix of the top bidders are allocated, but their prices equalize to (roughly) the lowest bid in the prefix (as in the example above).

2.2 The Price-Setting Mechanism. An equilibrium allocation of GFP can be computed directly via the following mechanism, which we refer to as the price-setting (PS) mechanism. Essentially this is a descending price mechanism: the price stops descending when the bidders willing to pay at that price have enough budget to purchase all the clicks. We have to be careful at the moment a bidder is added to the pool of the willing bidders; if this new bidder has a large enough budget, then suddenly the willing bidders have more than enough budget to pay for all of the clicks. To compensate, the mechanism decreases this 
"threshold" bidder's effective budget until the clicks are paid for exactly. We formalize the mechanism as follows:

\begin{tabular}{|l|}
\hline Price-Setting (PS) Mechanism (Single Slot) \\
\hline - Assume wlog that $b_{1} \succ b_{2} \succ \ldots \succ b_{n} \geq 0$. \\
- Let $k$ be the first bidder such that $b_{k+1} \leq \sum_{i=1}^{k} B_{i} / D$. Compute price \\
$p=\min \left\{\sum_{i=1}^{k} B_{i} / D, b_{k}\right\}$. \\
- Allocate $B_{i} / p$ clicks to each $i \leq k-1$. Allocate $\hat{B}_{k} / p$ clicks to bidder $k$, \\
where $\hat{B}_{k}=p D-\sum_{i=1}^{k-1} B_{i}$. \\
\hline
\end{tabular}

Example 2. Suppose there are three bidders with $b_{1}=\$ 2, b_{2}=\$ 1, b_{3}=\$ 0.25$ and $B_{1}=\$ 100, B_{2}=\$ 50, B_{3}=\$ 80$, and $D=300$ clicks. Running the PS mechanism, we get $k=2$ since $B_{1} / D=1 / 3<b_{2}=\$ 1$, but $\left(B_{1}+B_{2}\right) / D=\$ 0.50 \geq b_{3}=\$ 0.25$. The price is set to $\min \{\$ 0.50, \$ 1\}=\$ 0.50$, and bidders 1 and 2 get 200 and 100 clicks at that price, respectively. There is no threshold bidder.

Example 3. Suppose now bidder 2 changes her bid to $b_{2}=\$ 0.40$ (everything else remains the same as Example 2). We still get $k=2$ since $B_{1} / D=1 / 3<b_{2}=\$ 0.40$. But now the price is set to $\min \{\$ 0.50, \$ 0.40\}=\$ 0.40$, and bidders 1 and 2 get 250 and 50 clicks at that price, respectively. Note that bidder 2 is now a threshold bidder, does not use her entire budget, and gets fewer clicks.

Note that this mechanism reduces to the given mechanisms in the special cases of infinite bids or budgets (with the proper treatment of infinite bids/budgets).

Theorem 1. The price-setting mechanism (single slot) is truthful.

2.3 Price-Setting Mechanism Computes Nash Equilibrium of GFP. Consider the greedy first-price auction in which the highest bidder receives $B_{1} / b_{1}$ clicks, the second $B_{2} / b_{2}$ clicks and so on, until the supply of $D$ clicks is exhausted. It is immediate that truthfully reporting budgets is a dominant strategy in this mechanism, since when a bidder is considered, her reported budget is exhausted as much as possible, at a fixed price. However, reporting $b_{i}=m_{i}$ is not a dominant strategy. Nevertheless, it turns out that GFP has an equilibrium whose outcome is (roughly) the same as the PS mechanism. One cannot show that there is a plain Nash equilibrium because of the way ties are resolved lexicographically; the following example illustrates why.

Example 4. Suppose we have the same instance as example 1: two bidders, $D=120$ clicks, $\left(m_{1}=\$ 2, B_{1}=\$ 100\right)$ and $\left(m_{2}=\$ 1, B_{2}=\$ 50\right)$. But now suppose that bidder 2 occurs first lexicographically. In GFP, if bidder 2 tells the truth, and bidder 1 declares $b_{1}=\$ 1$, then bidder 2 will get chosen first (since she is first lexicographically), and take 50 clicks. Bidder 2 will end up with the remaining 70 clicks. However, if bidder 1 instead declares $b_{1}=\$ 1+\epsilon$ for some $\epsilon>0$, then she gets $100 /(1+\epsilon)$ clicks. But this is not a best response, since she could bid $1+\epsilon / 2$ and get slightly more clicks.

Thus, we prove instead that the bidders reach an $\epsilon$-Nash equilibrium: 
Theorem 2. Suppose the PS mechanism is run on the truthful input, resulting in price $p$ and clicks $c_{1}, \ldots, c_{n}$ for each bidder. Then, for any $\epsilon>0$ there is a pure-strategy $\epsilon$-Nash equilibrium of the GFP mechanism where each bidder receives $c_{i} \pm \epsilon$ clicks.

\section{Multiple Slots: Bids or Budgets Only}

Generalizing to multiple slots makes the scheduling constraint nontrivial. Now instead of splitting a pool of $D$ clicks arbitrarily, we need to assign clicks that correspond to a feasible schedule of bidders to slots. The conditions under which this is possible add a complexity that we characterize and incorporate into our mechanism in this section.

As in the single-slot case it will be instructive to consider first the cases of infinite bids or budgets. Suppose all $B_{i}=\infty$. In this case, the input consists of bids only $b_{1} \succ b_{2} \succ \ldots \succ b_{n}$. Naturally, what we do here is rank by bid, and allocate the slots to the bidders in that order. Since each budget is infinite, we can always set the prices $p_{i}$ equal to the bids $b_{i}$. By the same logic as in the single-slot case, this is easily seen to be truthful. In the other case, when $b_{i}=\infty$, there is a lot more work to do, and we devote the remainder of the section to this case.

Without loss of generality, we may assume the number of slots equals the number of bids (i.e., $n^{\prime}=n$ ); if this is not the case, then we add dummy bidders with $B_{i}=b_{i}=0$, or dummy slots with $D_{i}=0$, as appropriate. We keep this assumption for the remainder of the paper. The proofs of the theorems in this section are in Appendix B.

3.1 Assigning slots using a classical scheduling algorithm. First we give an important lemma that characterizes the conditions under which a set of bidders can be allocated to a set of slots, which turns out to be just a restatement of a classical result [14] from scheduling theory.

Lemma 1. Suppose we would like to assign an arbitrary set $\{1, \ldots, k\}$ of bidders to a set of slots $\{1, \ldots, k\}$ with $D_{1}>\ldots>D_{k}$. Then, a click allocation $c_{1} \geq$ $\ldots \geq c_{k}$ is feasible iff

$$
c_{1}+\ldots+c_{\ell} \leq D_{1}+\ldots+D_{\ell} \quad \text { for all } \ell=1, \ldots, k .
$$

Proof. In scheduling theory, we say a job with service requirement $x$ is a task that needs $x / s$ units of time to complete on a machine with speed $s$. The question of whether there is a feasible allocation is equivalent to the following scheduling problem: Given $k$ jobs with service requirements $x_{i}=c_{i}$, and $k$ machines with speeds $s_{i}=D_{i}$, is there a schedule of jobs to machines (with preemption allowed) that completes in one unit of time?

As shown in [14], the optimal schedule for this problem (a.k.a. $Q|p m t n| C_{\max }$ ) can be found efficiently by the level algorithm, ${ }^{5}$ and the schedule completes in time $\max _{\ell \leq k}\left\{\sum_{i=1}^{\ell} x_{i} / \sum_{i=1}^{\ell} s_{i}\right\}$. Thus, the conditions of the lemma are exactly the conditions under which the schedule completes in one unit of time.

\footnotetext{
${ }^{5}$ In later work, Gonzalez and Sahni [12] give a faster (linear-time) algorithm.
} 
3.2 A multiple-slot budgets-only mechanism. Our mechanism will roughly be a descending-price mechanism where we decrease the price until a prefix of budgets fits tightly into a prefix of positions at that price, whereupon we allocate that prefix, and continue to decrease the price for the remaining bidders.

The following subroutine, which will be used in our mechanism (and later in the general mechanism), takes a set of budgets and determines a prefix of positions that can be packed tightly with the largest budgets at a uniform price $p$. The routine ensures that all the clicks in those positions are sold at price $p$, and all the allocated bidders spend their budget exactly.

\begin{tabular}{|l|}
\hline Routine "Find-Price-Block" \\
\hline Input: Set of $n$ bidders, set of $n$ slots with $D_{1}>D_{2}>\ldots>D_{n}$. \\
- If all $D_{i}=0$, assign bidders to slots arbitrarily and exit. \\
- Sort the bidders by budget and assume wlog that $B_{1} \geq B_{2} \geq \ldots \geq B_{n}$. \\
- Define $r_{\ell}=\sum_{i=1}^{\ell} B_{i} / \sum_{i=1}^{\ell} D_{i}$. Set price $p=\max _{\ell} r_{\ell}$. \\
- Let $\ell^{*}$ be the largest $\ell$ such that $r_{\ell}=p$. Allocate slots $\left\{1, \ldots \ell^{*}\right\}$ to \\
bidders $\left\{1, \ldots, \ell^{*}\right\}$ at price $p$, using all of their budgets; i.e., $c_{i}:=B_{i} / p$. \\
\hline
\end{tabular}

Note that in the last step the allocation is always possible since for all $\ell \leq \ell^{*}$, we have $p \geq r_{\ell}=\sum_{i=1}^{\ell} B_{i} / \sum_{i=1}^{\ell} D_{i}$, which rewritten is $\sum_{i=1}^{\ell} c_{i} \leq \sum_{i=1}^{\ell} D_{i}$, and so we can apply Lemma 1 . Now we are ready to give the mechanism in terms of this subroutine; an example run is shown in Figure 1.

\section{Price-Setting Mechanism (Multiple Slots, Budgets Only)}

- Run "Find-Price-Block" on bidders $1, \ldots, n$, and slots $1, \ldots, n$. This gives an allocation of $\ell^{*}$ bidders to the first $\ell^{*}$ slots.

- Repeat on the remaining bidders and slots until all slots are allocated.

Let $p_{1}, p_{2}, \ldots$ be the prices used for each successive block assigned by the algorithm. We claim that $p_{1}>p_{2}>\ldots$; to see this, note then when $p_{1}$ is set, we have $p_{1}=r_{k}$ and $p_{1}>r_{\ell}$ for all $\ell>k$, where $k$ is the last bidder in the block. Thus for all $\ell>k$, we have $p_{1} \sum_{j<\ell} D_{j}>\sum_{i<\ell} B_{j}$, which gives $p_{1} \sum_{k<j \leq \ell} D_{j}>\sum_{k<i \leq \ell} B_{j}$ using $p_{1}=r_{k}$. This implies that when we apply Find-Price-Block the second time, we get $r_{\ell}^{\prime}=\sum_{k<i<\ell} B_{j} / \sum_{k<j \leq \ell} D_{j}<p_{1}$, and so $p_{2}<p_{1}$. This argument applies to successive blocks to give $p_{1}>p_{2}>\ldots$

Theorem 3. The price-setting mechanism (multiple slots, budgets only) is truthful.

\section{Main Results}

In this section we give our main results, presenting our price-setting mechanism in the general case, building on the ideas in the previous two sections. We begin in Section 4.1 by stating the mechanism and showing some examples, then proving that the mechanism is truthful. In Section 4.2 we analyze the revenue-optimal 


\begin{tabular}{|c|c|}
\hline Bidder & Budget \\
\hline 1 & $\$ 80$ \\
\hline 2 & $\$ 70$ \\
\hline 3 & $\$ 20$ \\
\hline 4 & $\$ 1$ \\
\hline
\end{tabular}

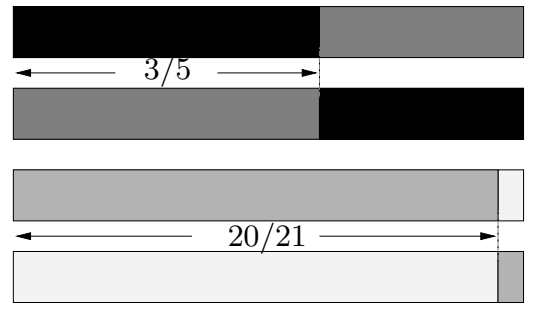

$$
\left.\begin{array}{l}
D_{1}=100 \\
D_{2}=50 \\
D_{3}=25 \\
D_{4}=0
\end{array}\right] p_{1}=\$ 1.00
$$

Fig. 1. An example of the PS mechanism (multiple slots, budgets only). We have four slots with $D_{1}, \ldots, D_{4}$ clicks as shown, and four bidders with declared budgets as shown. The first application of Find-Price-Block computes $r_{1}=B_{1} / D_{1}=80 / 100$, $r_{2}=\left(B_{1}+B_{2}\right) /\left(D_{1}+D_{2}\right)=150 / 150, r_{3}=\left(B_{1}+B_{2}+B_{3}\right) /\left(D_{1}+D_{2}+D_{3}\right)=170 / 175$, $r_{4}=\left(B_{1}+B_{2}+B_{3}+B_{4}\right) /\left(D_{1}+D_{2}+D_{3}+D_{4}\right)=171 / 175$. Since $r_{2}$ is largest, the top two slots make up the first price block with a price $p_{1}=r_{2}=\$ 1$; bidder 1 gets 80 clicks and bidder 2 gets 70 clicks, using the schedule as shown. In the second price block, we get $B_{3} / D_{3}=20 / 25$ and $\left(B_{3}+B_{4}\right) /\left(D_{3}+D_{4}\right)=21 / 25$. Thus $p_{2}$ is set to $21 / 25=\$ 0.84$, bidder 3 gets $500 / 21$ clicks and bidder 4 gets $25 / 21$ clicks, using the schedule as shown.

schedule, and show that it can be computed with a generalization of the greedy first-price (GFP) mechanism. We then show that GFP has an $\epsilon$-Nash equilibrium whose outcome is identical to the general PS mechanism. The proofs of the theorems in this section are in Appendix C.

4.1 The Price-Setting Mechanism (General Case). The generalization of the PS mechanism combines the ideas from the bids-and-budgets version of the single slot mechanism with the budgets-only version of the multiple-slot mechanism. As our price descends, we maintain a set of "active" bidders with bids at or above this price, as in the single-slot mechanism. These active bidders are kept ranked by budget, and when the price reaches the point where a prefix of bidders fits into a prefix of slots (as in the budgets-only mechanism) we allocate them and repeat. As in the single-slot case, we heave to be careful when a bidder enters the active set and suddenly causes an over-fit; in this case we again reduce the budget of this "threshold" bidder until it fits. We formalize this as follows:

\section{Price-Setting Mechanism (General Case)}

(i) Assume wlog that $b_{1} \succ b_{2} \succ \ldots \succ b_{n}=0$.

(ii) Let $k$ be the first bidder such that running Find-Price-Block on bidders $1, \ldots, k$ would result in a price $p \geq b_{k+1}$.

(iii) Reduce $B_{k}$ until running Find-Price-Block on bidders $1, \ldots, k$ would result in a price $p \leq b_{k}$. Apply this allocation, which for some $\ell^{*} \leq k$ gives the first $\ell^{*}$ slots to the $\ell^{*}$ bidders among $1 \ldots k$ with the largest budgets.

(iv) Repeat on the remaining bidders and slots.

An example run of this mechanism is shown in Figure 2. Since the PS mechanism sets prices per slot, it is natural to ask if these prices constitute some sort of 
"market-clearing" equilibrium in the spirit of a Fisher market. The quick answer is no: since the price per click increases for higher slots, and each bidder values clicks at each slot equally, then bidders will always prefer the bottom slot. Note that by the same logic as the budgets-only mechanism, the prices $p_{1}, p_{2}, \ldots$ for each price block strictly decrease.

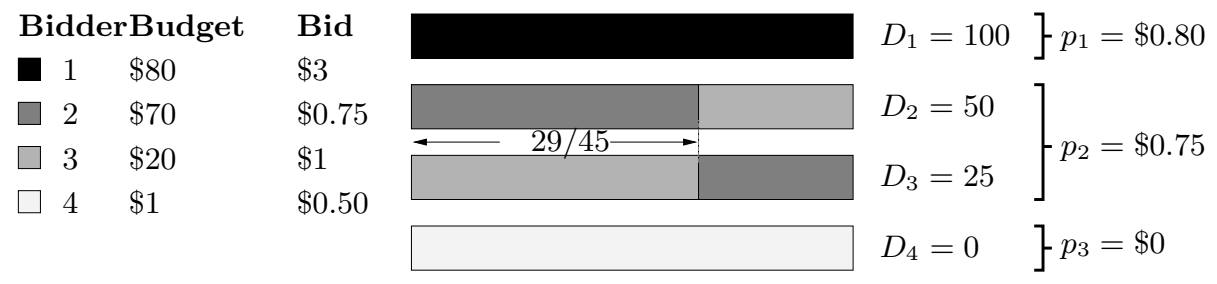

Fig. 2. Consider the same bidders and slots as in Figure 1, but now add bids as shown. Running Find-Price-Block on only bidder 1 gives a price of $r_{1}=80 / 100$, which is less than the next bid of $\$ 1$. So, we run Find-Price-Block on bidders 1 and 3 (the nexthighest bid), giving $r_{1}=80 / 100$ and $r_{2}=100 / 150$. We still get a price of $\$ 0.80$, but now this is more than the next-highest bid of $\$ 0.75$, so we allocate the first bidder to the first slot at a price of $\$ 0.80$. We are left with bidders $2-4$ and slots $2-4$. With just bidder 3 (the highest bidder) and slot 2 , we get a price $p=20 / 50$ which is less than the next-highest bid of $\$ 0.75$, so we consider bidders 2 and 3 on slots 2 and 3 . This gives a price of $\max \{70 / 50,90 / 75\}=\$ 1.40$, which is more than $\$ 0.50$. Since this is also more than $\$ 0.75$, we must lower $B_{2}$ until the price is exactly $\$ 0.75$, which makes $B_{2}^{\prime}=\$ 36.25$. With this setting of $B_{2}^{\prime}$, Find-Price-Block allocates bidders 2 and 3 to slots 2 and 3, giving $75(36.25 / 56.25)$ and $75(20 / 56.25)$ clicks respectively, at a price of $\$ 0.75$ per click. Bidder 4 is allocated to slot 4 , receiving zero clicks.

Efficiency. So far we have been largely ignoring the efficiency of computing the allocation in the PS mechanism. It is immediately clear that the general PS mechanism can be executed in time polynomial in $n$ and $\log (1 / \epsilon)$ to some precision $\epsilon$ using binary search and linear programming.

In fact, a purely combinatorial $O\left(n^{2}\right)$ time algorithm is possible. As bidders get added in step (ii), maintaining a sorted list of bidders and budgets can be done in time $O(n \log n)$. Thus it remains to show that running Find-Price-Block (and computing the reduced budget) can be done in $O(n)$ time given these sorted lists. In Find-Price-Block, computing the ratios $r_{\ell}$ can be done in linear time. Finding the allocation from Lemma 1 can also be done in linear time using the Gonzalez-Sahni algorithm [12] for scheduling related parallel machines (in fact the total time for scheduling can be made $O(n)$ since each slot is scheduled only once). Finally, computing the reduced budget is a simple calculation on each relevant ratio $r_{\ell}$, also doable in linear time. We suspect that there is a $O(n \cdot \operatorname{poly} \log (n))$ algorithm using a more elaborate data structure; we leave this open.

Theorem 4. The price-setting mechanism (general case) is truthful. 
4.2 Greedy First-Price Mechanism for Multiple Slots. In the general case, as in the single-slot case, there is a natural greedy first-price mechanism when the bidding language includes both bids and budgets: Order the bidders by bid $b_{1} \succ b_{2} \succ \ldots \succ b_{n}$. Starting from the highest bidder, for each bidder $i$ compute the maximum possible number of clicks $c_{i}$ that one could allocate to bidder $i$ at price $b_{i}$, given the budget constraint $B_{i}$ and the commitments to previous bidders $c_{1}, \ldots, c_{i-1}$. This reduces to the "fractional knapsack" problem in the single-slot case, and so one would hope that it maximizes revenue for the given bids and budgets, as in the single-slot case. This is not immediately clear, but does turn out to be true, as we will prove in this section.

As in the single-slot case, the greedy mechanism is not a truthful mechanism. However, we show that it does have a pure-strategy equilibrium, and that equilibrium has prices and allocation equivalent to the price setting mechanism.

Greedy is Revenue-Maximizing. Consider a revenue-maximizing schedule that respects both bids and budgets. In this allocation, we can assume wlog that each bidder $i$ is charged exactly $b_{i}$ per click, since otherwise the allocation can increase the price for bidder $i$, reduce $c_{i}$ and remain feasible. Thus, by Lemma 1 , we can find a revenue-maximizing schedule $\mathbf{c}^{*}=\left(c_{1}^{*}, \ldots, c_{n}^{*}\right)$ by maximizing $\sum_{i} b_{i} c_{i}$ subject to $c_{i} \leq B_{i} / b_{i}$ and $c_{1}+\ldots+c_{\ell} \leq D_{1}+\ldots+D_{\ell}$ for all $\ell=1, \ldots, n$.

Theorem 5. The greedy first-price auction gives a revenue-maximizing schedule.

Price-Setting Mechanism is a Nash Equilibrium of the Greedy First Price Mechanism. We note that truthfully reporting one's budget is a weakly dominant strategy in GFP, since when a bidder is considered for allocation, their budget is exhausted at a fixed price, subject to a cap on the number of clicks they can get. Reporting one's bid truthfully is not a dominant strategy, but we can still show that there is an $\epsilon$-Nash equilibrium whose outcome is arbitrarily close to the PS mechanism.

Theorem 6. Suppose the PS mechanism is run on the truthful input, resulting in clicks $c_{1}, \ldots, c_{n}$ for each bidder. Then, for any $\epsilon>0$ there is a pure-strategy $\epsilon$-Nash equilibrium of the GFP mechanism where each bidder receives $c_{i} \pm \epsilon$ clicks.

\section{Conclusions}

In this paper we have given a truthful mechanism for assigning bidders to clickgenerating slots that respects budget and per-click price constraints. The mechanism also respects a scheduling constraint on the slots, using a classical result from scheduling theory to characterize (and compute) the possible allocations. We have also proved that the revenue-maximizing mechanism has an $\epsilon$-Nash equilibrium whose outcome is arbitrarily close to our mechanism. This final result in some way suggests that our mechanism is the right one for this model. It would interesting to make this more formal; we conjecture that a general truthful mechanism cannot do better in terms of revenue. 
5.1 Extensions. There are several natural generalizations of the Online Ad Slot Scheduling problem where it would be interesting to extend our results or apply the knowledge gained in this paper. We mention a few here.

Click-through rates. In sponsored search (e.g. [9]) it is common for each bidder to have a personal click-through-rate $\gamma_{i}$; in our model this would mean that a bidder $i$ assigned to slot $j$ for a time period of length $\alpha$ would receive $\alpha \gamma_{i} D_{j}$ clicks. All our results can be generalized to this setting by simply scaling the bids using $b_{i}^{\prime}=b_{i} \gamma_{i}$. However, our mechanism in this case does not necessarily prefer more efficient solutions; i.e., ones that generate more overall clicks. It would be interesting to analyze a possible tradeoff between efficiency and revenue in this setting.

Multiple Keywords. To model multiple keywords in our model, we could say that each query $q$ had its own set of click totals $D_{q, 1} \ldots D_{q, n}$, and each bidder is interested in a subset of queries. The greedy first-price mechanism is easily generalized to this case: maximally allocate clicks to bidders in order of their bid $b_{i}$ (at price $b_{i}$ ) while respecting the budgets, the query preferences, and the click commitments to previous bidders. It would not be surprising if there was an equilibrium of this extension of the greedy mechanism that could be computed directly with a generalization of the PS mechanism.

Online queries, uncertain supply. In sponsored search, allocations must be made online in response to user queries, and some of the previous literature has focused on this aspect of the problem (e.g., $[20,19])$. Perhaps the ideas in this paper could be used to help make online allocation decisions using (unreliable) estimates of the supply, a setting considered in [19], with game-theoretic considerations.

Acknowledgments. We thank Cliff Stein and Yishay Mansour for helpful discussions.

\section{References}

1. Zoë Abrams, Ofer Mendelevitch, and John Tomlin. Optimal delivery of sponsored search advertisements subject to budget constraints. In ACM Conference on Electronic Commerce, pages 272-278, 2007.

2. Gagan Aggarwal, Ashish Goel, and Rajeev Motwani. Truthful auctions for pricing search keywords. In EC '06: Proceedings of the 7th ACM conference on Electronic commerce, pages 1-7, New York, NY, USA, 2006. ACM.

3. Kenneth J. Arrow and Gerard Debreu. Existence of an equilibrium for a competitive economy. Econometrica, 22(3):265-290, July 1954.

4. Christian Borgs, Jennifer Chayes, Nicole Immorlica, Mohammad Mahdian, and Amin Saberi. Multi-unit auctions with budget-constrained bidders. In $E C$ '05: Proceedings of the 6th ACM conference on Electronic commerce, pages 44-51, New York, NY, USA, 2005. ACM.

5. Christian Borgs, Jennifer T. Chayes, Nicole Immorlica, Kamal Jain, Omid Etesami, and Mohammad Mahdian. Dynamics of bid optimization in online advertisement auctions. In Carey L. Williamson, Mary Ellen Zurko, Peter F. Patel-Schneider, and Prashant J. Shenoy, editors, WWW, pages 531-540. ACM, 2007. 
6. Matthew Cary, Aparna Das, Benjamin Edelman, Ioannis Giotis, Kurtis Heimerl, Anna R. Karlin, Claire Mathieu, and Michael Schwarz. Greedy bidding strategies for keyword auctions. In ACM Conference on Electronic Commerce, pages 262-271, 2007.

7. Xiaotie Deng, Christos Papadimitriou, and Shmuel Safra. On the complexity of equilibria. In STOC '02: Proceedings of the thiry-fourth annual ACM symposium on Theory of computing, pages 67-71, New York, NY, USA, 2002. ACM.

8. Nikhil R. Devanur, Christos H. Papadimitriou, Amin Saberi, and Vijay V. Vazirani. Market equilibrium via a primal-dual-type algorithm. In FOCS '02: Proceedings of the 43rd Symposium on Foundations of Computer Science, pages 389-395, Washington, DC, USA, 2002. IEEE Computer Society.

9. Benjamin Edelman, Michael Ostrovsky, and Michael Schwarz. Internet advertising and the generalized second-price auction: Selling billions of dollars worth of keywords. American Economic Review, 97(1):242-259, March 2007. available at http://ideas.repec.org/a/aea/aecrev/v97y2007i1p242-259.html.

10. Edmund Eisenberg and David Gale. Consensus of subjective probabilities: The pari-mutuel method. Annals Of Mathematical Statistics, 30(165):165-168, March 1959.

11. Jon Feldman, S Muthukrishnan, Martin Pal, and Cliff Stein. Budget optimization in search-based advertising auctions. In EC 'O\%: Proceedings of the 8th ACM conference on Electronic commerce, pages 40-49, New York, NY, USA, 2007. ACM.

12. Teofilo Gonzalez and Sartaj Sahni. Preemptive scheduling of uniform processor systems. J. ACM, 25(1):92-101, 1978.

13. R.L. Graham, E.L. Lawler, J.K. Lenstra, and A.H.G. Rinnooy Kan. Optimization and approximation in deterministic sequencing and scheduling: a survey. Ann. Discrete Math., 4:287-326, 1979.

14. Edward C. Horvath, Shui Lam, and Ravi Sethi. A level algorithm for preemptive scheduling. J. ACM, 24(1):32-43, 1977.

15. Ramesh Johari and John N. Tsitsiklis. Efficiency loss in a network resource allocation game. Math. Oper. Res., 29(3):407-435, 2004.

16. Frank P. Kelly. Charging and rate control for elastic traffic. European Transactions on Telecommunications, 8:33-37, January 1997.

17. Sébastien Lahaie. An analysis of alternative slot auction designs for sponsored search. In EC '06: Proceedings of the 7th ACM conference on Electronic commerce, pages 218-227, New York, NY, USA, 2006. ACM.

18. Sébastien Lahie, David Pennock, Amin Saberi, and Rakesh Vohra. Sponsored Search Auctions, in: Algorithmic Game Theory, pages 699-716. Cambirdge University Press, 2007.

19. Mohammad Mahdian, Hamid Nazerzadeh, and Amin Saberi. Allocating online advertisement space with unreliable estimates. In ACM Conference on Electronic Commerce, pages 288-294, 2007.

20. Aranyak Mehta, Amin Saberi, Umesh Vazirani, and Vijay Vazirani. Adwords and generalized on-line matching. In FOCS '05: Proceedings of the 46th Annual IEEE Symposium on Foundations of Computer Science, pages 264-273, Washington, DC, USA, 2005. IEEE Computer Society.

21. Paat Rusmevichientong and David P. Williamson. An adaptive algorithm for selecting profitable keywords for search-based advertising services. In EC '06: Proceedings of the 7th ACM conference on Electronic commerce, pages 260-269, New York, NY, USA, 2006. ACM.

22. Hal Varian. Position auctions. International Journal of Industrial Organization, 25(6):1163-1178, December 2007. 


\section{A Proofs for Section 2}

Proof of Theorem 1: For the purposes of this proof, let bidders $\{1, \ldots, n\}$ be such that $b_{1} \succ \ldots \succ b_{n}=0$, and consider a new bidder (call her Alice) with true max-cpc $m$ and true budget $B^{*}$.

We first show that reporting the true budget is a weakly dominant strategy for Alice, for any fixed bid $b>0$. Let $\ell$ be the first bidder with $b \succ b_{\ell}$, so $b_{1} \succ \ldots \succ b_{\ell-1} \succ b \succ b_{\ell} \succ \ldots \succ b_{n}$. Let $\mathcal{B}=\sum_{i=1}^{\ell-1} B_{i}$. If $\mathcal{B} \geq b D$ then the mechanism will not allocate any clicks to Alice, regardless of the reported budget, since the price will stop before reaching $b$. If $\mathcal{B}<b D$, we will argue that Alice's clicks $c$ are non-increasing in $B$. Define $\hat{B}=b D-\mathcal{B}>0$.

- If Alice declares $B \in[\hat{B}, \infty]$, then the price will stop at $b$. She will spend $\hat{B}$ and receive $c=\hat{B} / b$ clicks.

- If Alice declares $B \in[0, \hat{B})$, then the price will be lower than $b$, and she will spend all of her budget. Her final number of clicks will be $c=(B /(B+\mathcal{B}+R)) D$, where $R$ is the total spend of bidders $\{\ell, \ldots, n\}$. Since $R$ is non-increasing in $B$, we can conclude that $c$ is non-decreasing in $B$.

Putting together these intervals, we see that $c$ is non-decreasing in $B$ overall, and since Alice's total spend is $\min \{B, \hat{B}\}$, we may conclude that it is weakly dominant to declare $B=B^{*}$.

It remains to show that it is weakly dominant for Alice to declare a bid $b=m$, given that she declares a budget $B=B^{*}$. Let $R(b)$ be the total spend of bidders $\{1, \ldots, n\}$ given that Alice declares $b$. Note that $R(b)$ is non-increasing in $b$. Let $p_{1}$ be the price that would result if $b=\infty$, and let $p_{2}$ be the price that would result if $b=0$. Note that $p_{2} \leq p_{1}$.

- If $b \in\left[0, p_{2}\right)$ then the price stops at $p_{2}$ and Alice receives zero clicks.

- If $b \in\left(p_{1}, \infty\right]$, then the price stops at $p_{1}$, and Alice receives $B / p_{1}$ clicks.

- If $b \in\left[p_{2}, p_{1}\right]$, then the price stops at $b$. To see this, note that if Alice had bid zero, then the price would have gone down to $p_{2}$, so it certainly stops at $b$ or lower. But at price $b$, the set of bidders that can afford this price consists of at least all the bidders that could afford price $p_{1}$, and so we must have $B+\sum_{i: b_{i} \succ b} B_{i} \geq B+\sum_{i: b_{i} \geq p_{1}} B_{i} \geq p_{1} D \geq b D$. Alice thus receives

$$
\max \left\{0, D-\left(\sum_{i: b_{i} \succ b} B_{i} / b\right)\right\}
$$

clicks, and we may conclude that in this interval, clicks are non-decreasing with $b$.

Note that in the expression (2), plugging in $p_{1}$ for $b$ yields $c=B / p_{1}$. Thus we have that in the interval $\left[p_{2}, \infty\right]$, clicks are non-decreasing with $b$, and the price is always $\min \left\{b, p_{1}\right\}$. We conclude that bidding $b=m$ is a weakly dominant strategy. 
Proof of Theorem 2: We will show that for sufficiently small $\epsilon^{\prime}>0$, if each bidder truthfully reports her budget and bids $b_{i}=\min \left\{m_{i}, p+\epsilon^{\prime}\right\}$ in the GFP mechanism, then the conditions in the theorem hold.

There are two ways that the PS mechanism (under truthful input) can reach its last allocated bidder $k$ and final price $p$ : if $m_{k}>p \geq m_{k+1}$ and then $p D=$ $\sum_{i=1}^{k} B_{i}$ (no threshold bidder), or if $p=m_{k}$ ( $k$ is a threshold bidder).

In the first case, we have that bidders $i \leq k$ all have $m_{i}>p$. Thus in the supposed equilibrium of GFP, all these bidders are bidding $p+\epsilon^{\prime}$, and all bidders $i>k$ are bidding $m_{i} \leq p$. Therefore in GFP, each $i \leq k$ will receive $B_{i} /\left(p+\epsilon^{\prime}\right)$ clicks, and the total number of clicks allocated by GFP to bidders $1 \ldots k$ is $\sum_{i \leq k} B_{i} /\left(p+\epsilon^{\prime}\right)=\left(\frac{p}{p+\epsilon^{\prime}}\right) D$. The remaining $D^{\prime}=\left(1-\frac{p}{p+\epsilon}\right) D$ clicks, are allocated to bidders $i>k$. Bidders $1 \ldots k$ lose clicks by increasing their bid, and can gain at most $D^{\prime}$ clicks by lowering their bid. Bidders $i>k$ will never raise their bid (since they are bidding $m_{i}$ ), and cannot gain more clicks by lowering their bid. Since $D^{\prime}$ can be made arbitrarily small, we have an $\epsilon$-Nash equilibrium.

In the second case, $p=m_{k}$. Let $k^{\prime}<k$ be the last bidder bidding more than $p$. In the supposed GFP equilibrium, bidders $1 \ldots k^{\prime}$ are bidding $p+\epsilon^{\prime}$, and bidders $\left(k^{\prime}+1, \ldots, k\right)$ are bidding $m_{k}=p$. Thus GFP allocates $B_{i} /\left(p+\epsilon^{\prime}\right)$ clicks to bidders $1 \ldots k^{\prime}, B_{i} / p$ clicks to bidders $\left(k^{\prime}+1, \ldots, k-1\right)$ (if any such bidders exist) and the remaining clicks to bidder $k$. As in the previous case, no bidder can gain from raising her bid, the number of clicks that a bidder $i \leq k^{\prime}$ can gain from lowering her bid can be made arbitrarily small, and no other bidder can gain from lowering her bid.

\section{B $\quad$ Proofs for Section 3}

Lemma 2. In Find-Price-Block, if $B_{i}=B_{i+1}$, then $i$ cannot be the last slot of the computed price block.

Proof. Suppose the contrary, namely that $i$ is the last slot of the first price block and $(i+1)$ is the first slot in the second price block. Denote $B=B_{1}+\ldots+B_{i-1}$ and $D=D_{1}+\ldots+D_{i-1}$. Then the price of the first price block satisfies (1) $p_{1}=$ $\frac{B+B_{i}}{D+D_{i}} \geq \frac{B}{D}$ and $(2) p_{1}=\frac{B+B_{i}}{D+D_{i}}>\frac{B+B_{i}+B_{i+1}}{D+D_{i}+D_{i+1}}$. The first condition is equivalent to $\frac{B_{i}}{D_{i}} \geq \frac{B+B_{i}}{D+D_{i}}$, and the second condition is equivalent to $\frac{B+B_{i}}{D+D_{i}}>\frac{B_{i+1}}{D_{i+1}}$. The latter two inequalities imply $\frac{B_{i}}{D_{i}}>\frac{B_{i+1}}{D_{i+1}}$, which is a contradiction to the fact that $B_{i}=B_{i+1}$ and $D_{i}>D_{i+1}$.

Proof Sketch of Theorem 3: Suppose bidders $1, \ldots, n$ declare budgets $B_{1} \geq \ldots \geq$ $B_{n}$, and Alice declares budget $B$. Let $\ell_{B}$ be the rank of Alice by budget (and lexicographic order in case of ties) if she bids $B$. We will prove that the number of clicks Alice receives is non-increasing as she lowers her declared budget $B$, which immediately implies that truthful reporting of budgets is weakly dominant in the PS mechanism.

Let $r_{j}^{B}$ be the ratio $r_{j}$ assuming Alice bids $B$; so $r_{k}^{B}=\left(B+\sum_{i=1}^{k-1} B_{i}\right) / \sum_{i=1}^{k} D_{i}$ if $\ell_{B} \leq k$, and $r_{k}^{B}=\sum_{i=1}^{k} B_{i} / \sum_{i=1}^{k} D_{i}$ otherwise. For a declared budget $B$, 
let $k_{B}$ be the last slot in the first price block chosen by the mechanism. So, $k_{B}=\arg \max _{k} r_{k}^{B}$ (if there are multiple maxima, then $k_{B}$ is the largest lexicographically).

For sufficiently large $B>B_{1}$, we get that $r_{1}^{B}>r_{k}^{B}$ for all $k$ and so $k_{B}=1$. For any such $B$ Alice receives $D_{1}$ clicks, the most possible. Now as we lower $B$, two significant events could occur; we could drop to another bidder's budget $B_{i}$, or we could have a change in $k_{B}$, thus changing the set of bidders in the first block. If neither of these events occur, then Alice remains in the first price block, but gets a smaller share of the clicks. Thus it remains to cover these two events.

If $B=B_{i}$ for some $i$, then note that by Lemma 2 , Alice cannot be the last bidder in the block, so $i$ is in the same block as Alice. Therefore we may exchange the roles of Alice and bidder $i$ lexicographically (i.e., increase Alice's rank by one) and nothing changes.

Now suppose $B$ reaches a point where $r_{k}$ changes because $\arg \max _{k} r_{k}^{B}$ changes from $k_{B}$ to $k^{\prime}$. We use $k^{*}=k_{B}$ for the remainder of the proof for ease of notation. At the bid $B$ we have $r_{k^{*}}^{B}=r_{k^{\prime}}^{B}$. We claim that either $k^{\prime}>k^{*}$ or $k^{\prime}<\ell_{B}$. To see this note that for any $k$ between $\ell_{B}$ and $k^{*}$ we have that $r_{k}^{B}$ decreases at a rate of $1 /\left(\sum_{i=1}^{k} D_{i}\right)$, which is faster than the rate of the highest ratio $r_{k^{*}}^{B}$.

If $k^{\prime}>k^{*}$ then Alice remains in the first block, but it expands from ending at $k^{*}$ to ending at $k^{\prime}$. Both before and after the change in $r_{k}$, Alice is spending her entire budget at price $r_{k^{*}}^{B}=r_{k^{\prime}}^{B}$, so her clicks remain the same.

If $k^{\prime}<\ell_{B}$ then Alice would remain in a block ending at slot $k^{*}$, since $r_{k^{*}}^{B}$ remains maximum among $r_{\ell_{B}}^{B}, \ldots, r_{n}^{B}$ (by the same reasoning about "rate" as above). Since $r_{k^{*}}^{B}=r_{k^{\prime}}^{B}$ we have that the price of Alice's block and the first block will be the same. Since Alice is spending her entire budget before and after the change in $r_{k}$ at the same price, her clicks remain the same. As we continue to decrease $B$ beyond this point, we simply remove the bidders and slots from the first price block, and imagine that we are again in the first price block of a reduced instance.

\section{Proofs for Section 4}

Proof Sketch of Theorem 4: We split the proof into two lemmas, showing that clicks are non-decreasing in both bids and budgets. This immediately implies the theorem. First we need a small observation about Find-Price-Block:

Lemma 3. Suppose Find-Price-Block is run on a set of budgets $B_{1} \geq \ldots \geq B_{n}$ and produces a block $1, \ldots, \ell^{*}$ with price $p$. Then if a bidder is added to the set with budget $B$, and Find-Price-Block still produces price $p$, we must have that $B \leq B_{\ell^{*}}$.

Proof. Suppose not. Then $B>B_{\ell *}$ and we have that $\left(B+\sum_{i=1}^{\ell^{*}-1} B_{i}\right) / \sum_{i=1}^{\ell^{*}} D_{i} \leq$ $p$. This contradicts $p=\sum_{i=1}^{\ell^{*}} B_{i} / \sum_{i=1}^{\ell^{*}} D_{i}$, since $B>B_{\ell *}$.

Lemma 4. The number of clicks a bidder is allocated is non-decreasing in her declared budget. 
Proof sketch: Let bidders $\{1, \ldots, n\}$ be such that $b_{1} \succ \ldots \succ b_{n}$, and consider a new bidder Alice with bid $b_{\ell-1} \succ b \succ b_{\ell}$. We will argue that the number of clicks that Alice receives is non-increasing as she reduces her declared budget $B$.

Suppose Alice declares $B=\infty$ and let $\hat{B}$ be the amount she would spend (Alice would always be a threshold bidder if she declared $B=\infty$ ). Any declared budget $B \in[\hat{B}, \infty]$ would result in the same number of clicks, because $B$ is reduced by the mechanism in step (iii) to $\hat{B}$.

Now as $B$ decreases from $\hat{B}$, two different events could occur: (a) Alice's price block threshold $\ell^{*}$ could change (because Find-Price-Block outputs a different $\ell^{*}$ ) or (b) the lowest bidder $k$ could change (because running Find-Price-Block on $1, \ldots, k$ gave a price less than $\left.b_{k+1}\right)$. For event (a), and between these events, the arguments from Theorem 3 imply that Alice's clicks are non-increasing.

For event (b), when the price of the Alice's block is exactly $b_{k+1}$, if bidder $k+1$ is added, the resulting price output by Find-Price-Block in step (ii) is still at least $b_{k+1}$, since adding a bidder cannot reduce the price. Also Lemmas 3 and 2 together imply that Alice is still in the price block chosen in step (iii). Thus Alice's clicks do not increase.

Lemma 5. The number of clicks a bidder is allocated is non-decreasing in her declared bid.

Proof sketch: For the purposes of this proof, let bidders $\{1, \ldots, n\}$ be such that $b_{1} \succ \ldots \succ b_{n}$, and consider a new bidder (call her Alice) with declared budget $B$. We will argue that the number of clicks that Alice receives in non-increasing with her declared bid $b$.

Let $p_{1}$ be the price that Alice would pay if $b=\infty$, and suppose Alice is in the $j$ th price block when she bids $\infty$. Note that for any bid $b \in\left(p_{1}, \infty\right]$, Alice is still in the $j$ th price block and receives the same number of clicks $\left(B / p_{1}\right)$. Let $p_{2}$ be the minimum bid required to keep Alice in the $j$ th price block.

We claim that if $b \in\left[p_{2}, p_{1}\right]$, the price will always be exactly $b$ : no allocation is made until Alice is considered in step (ii), and when she's considered, FindPrice-Block returns a price $p \geq p_{1}$, since the set of bidders considered contains all the bidders who produced price $p_{1}$. Thus Alice is a threshold bidder, and in step (iii) Alice's budget is reduced so that the price is exactly $b$.

Let $k_{b}$ be the number of bidders with bid $b_{i} \succ b$. Let $B_{i}^{b}$ be the $i$ th largest budget among bidders with bid $b_{i} \succ b$. We claim that if $b \in\left[p_{2}, p_{1}\right]$, we have $\sum_{i=1}^{\ell} B_{i}^{b} / \sum_{i=1}^{\ell} D_{i}<b$ for all $\ell \leq k_{b}$, since otherwise Alice would not be in the $j$ th block.

Let $\hat{B}_{b}$ be Alice's reduced budget when she bids $b \in\left[p_{2}, p_{1}\right]$, and let $c_{b}=\hat{B}_{b} / b$ denote the number of clicks she receives. To satisfy the price being at most $b$ in step (iii), we must have that for all $\ell \leq k_{b}, \hat{B}_{b} \leq B_{\ell}^{b}+\Delta$, where $\Delta>0$ satisfies $\left(\Delta+\sum_{i=1}^{\ell} B_{i}^{b}\right) / \sum_{i=1}^{\ell} D_{i}=b$. In addition, we must have $\left(B_{b}+\sum_{i=1}^{k_{b}} B_{i}^{b}\right) / \sum_{i=1}^{k_{b}+1} D_{i} \leq$ $b$. Putting these constraints together we get $\hat{B}_{b}=\min _{\ell \leq k_{b}+1}\left\{b \sum_{i=1}^{\ell} D_{i}-\sum_{i=1}^{\ell-1} B_{i}^{b}\right\}$ and so

$$
c_{b}=\hat{B}_{b} / b=\min _{\ell \leq k_{b}+1}\left\{\sum_{i=1}^{\ell} D_{i}-\frac{1}{b} \sum_{i=1}^{\ell-1} B_{i}^{b}\right\} .
$$


As $b$ decreases, if the set of bidders with bids $\succ b$ doesn't change, then the $B_{i}^{b} \mathrm{~s}$ don't change, and so this expression implies that $c_{b}$ also decreases. If $b$ decreases to the point where $b^{\prime} \succ b$ for some new bidder $b^{\prime}$, then we claim that $c_{b}$ also cannot increase. To see this note that for all $\ell$, the expression $\sum_{i=1}^{\ell-1} B_{i}^{b}$ can only increase or stay the same if a new bidder is added. We conclude that $c_{b}$ is non-increasing in the interval $b \in\left[p_{2}, p_{1}\right]$.

When $b$ decreases to $p_{2}$, we transition from Alice being in the $j$ th price block to the $j+1$ st price block. As in Theorem 3, at the point of transition the $j$ th price block will have the same price as the $j+1$ st price block, and in both scenarios Alice spends exactly $\hat{B}_{p_{2}}$. Thus her clicks do not change. We can iterate these arguments for the $j+1$ st price block, and so the theorem is proven.

Lemmas 4 and 5 immediately imply Theorem 4.

Proof of Theorem 5: Note that an equivalent statement of the constraint $c_{1}+$ $\ldots+c_{\ell} \leq D_{1}+\ldots+D_{\ell}$ for all $\ell=1, \ldots, n$. is:

$$
\sum_{i \in S} c_{i}^{\prime} \leq D_{1}+\ldots+D_{|S|} \quad \text { for all subsets } S \subseteq\{1, \ldots, n\} .
$$

Suppose bids are $b_{1} \succ b_{2} \succ \ldots \succ b_{n}$ and the corresponding clicks given to bidders in the greedy allocation are $\mathbf{c}=\left(c_{1}, \ldots, c_{n}\right)$. Let $\mathbf{c}^{*}=\left(c_{1}^{*}, \ldots, c_{n}^{*}\right)$ be the revenue-maximizing solution with the closest prefix to c, meaning that the first $i$ such that $c_{i} \neq c_{i}^{*}$ is maximized, and modulo that, $c_{i}-c_{i}^{*}$ is minimized.

We shall prove that the greedy $\mathbf{c}$ gives a revenue-maximizing schedule. Suppose the contrary and let $i$ be the first index on which $\mathbf{c}$ differs from $\mathbf{c}^{*}$. Note that $c_{i}>c_{i}^{*}$ (by the definition of greedy, $c_{i}$ is the maximum possible given $\left.c_{1}, \ldots, c_{i-1}\right)$. Let $c_{\max }^{*}=\max \left\{c_{i+1}^{*}, \ldots, c_{n}^{*}\right\}$. Let $J=\left\{j>i: c_{j}^{*}=c_{\max }^{*}\right\}$. Consider an arbitrary tight constraint on $\mathbf{c}^{*}$ of the form (3), defined by the set $S$. We claim that if $i \in S$, then all $j \in J$ are also in $S$.

Proof of claim: Suppose the contrary, namely that $i \in S$ and $j \notin S$ for some $j \in J$. Applying (3), we get

$$
\sum_{\ell \in S} c_{\ell}^{*}=\sum_{\ell \leq|S|} D_{\ell}
$$

One of the bidders in $S$ must have index $m>i$, otherwise (3) would be violated for $c$ and $S$ by $\sum_{\ell \in S \subseteq\{1, \ldots, i\}} c_{\ell}>\sum_{\ell \in S \subseteq\{1, \ldots, i\}} c_{\ell}^{*}=\sum_{\ell \leq|S|} D_{\ell}$. If $m \notin J$, then we would violate $(3)$ for the set $S^{\prime}=S \cup\{j\} \backslash\{m\}: \sum_{\ell \in S^{\prime}} c_{\ell}^{*}>\sum_{\ell \in S} c_{\ell}^{*}=$ $\sum_{\ell \leq|S|=\left|S^{\prime}\right|} D_{\ell}$. Therefore $m \in J$.

Now by the feasibility of $\mathbf{c}^{*}$ and the fact that $j \notin S$, we also have $c_{j}^{*}+$ $\sum_{\ell \in S} c_{\ell}^{*} \leq D_{|S|+1}+\sum_{\ell \leq|S|} D_{\ell}$ which implies, together with (4), that $c_{j}^{*} \leq$ $D_{|S|+1}$. Again by feasibility, we also have $\sum_{\ell \in S \backslash m} c_{\ell}^{*} \leq \sum_{\ell \leq|S|-1} D_{\ell}$ and this, together with (4), gives $c_{m}^{*} \geq D_{|S|}$. Putting these last two observations together yields $D_{|S|} \leq c_{m}=c_{\max }^{*}=c_{j}^{*} \leq D_{|S|+1}$. Unless $c_{m}=c_{\max }^{*}=c_{j}^{*}=0$, this violates the distinctness of the non-zero $D_{j}$ 's. But if $c_{\max }^{*}=0$, it means that all 
$c_{\ell}$ for $\ell>i$ have $c_{\ell}=0$, which means that $\mathbf{c}$ gives strictly more clicks than $\mathbf{c}^{*}$, a contradiction.

Let $j$ be an arbitrary member of $J$. By the claim, there is an $\epsilon>0$ such that if we set $\mathbf{c}^{\prime}=\mathbf{c}^{*}$ except $c_{i}^{\prime}=c_{i}^{*}+\epsilon$ and $c_{j}^{\prime}=c_{j}^{*}-\epsilon$, we get a feasible allocation $\mathbf{c}^{\prime}$, since $j$ appears in every tight constraint in which $i$ appears. This allocation has revenue at least that of $\mathbf{c}^{*}$, since $b_{i} \geq b_{j}$. But, it has a closer prefix to $\mathbf{c}$ than c $^{*}$, a contradiction.

Proof Sketch of Theorem 6: We will abuse notation and let $\epsilon^{\prime}$ denote any positive quantity that can be made arbitrarily close to zero. When the PS mechanism is run on the truthful input, let $p_{1}>p_{2}>\ldots$ denote the prices of each block. We will show that if in GFP each bidder $i$ truthfully reports her budget and bids $b_{i}=\min \left\{m_{i}, p_{j}+\epsilon^{\prime}\right\}$, where $j$ is the price block of $i$ in the PS mechanism, we meet the conditions of the theorem.

Suppose the first price block is determined when bidder $k$ is considered, and ends at slot $\ell^{*} \leq k$. The price $p_{1}$ satisfies $m_{k+1} \leq p_{1} \leq m_{k}$. Let $P \subseteq[k]$ denote the bidders in the first block (the ones in $[k]$ with the $\ell^{*}$ highest budgets). Also, we have that all $i \in P$ spend their entire budget in the PS mechanism, except possibly $k$, who may spend less than her budget if $m_{k}=p_{1}$. We now argue that GFP will produce the same allocation as the PS mechanism for this price block. For all $i \in P$ we have $b_{i}=\min \left\{m_{i}, p_{1}+\epsilon^{\prime}\right\} \geq \min \left\{m_{k}, p_{1}\right\}=p_{1}$. All bidders $i \in([k] \backslash P)$ have $b_{i} \leq p_{2}+\epsilon^{\prime}<p_{1}$. All bidders $i \notin[k]$ have $m_{i} \prec m_{k}$ and so since $b_{i} \leq m_{i}$ we get $b_{i} \prec b_{i^{\prime}}$ for all $i^{\prime} \in P$. We conclude that the bidders in $P$ are the first to be considered by the GFP mechanism. Furthermore, if $k \in P$, and $B_{k}$ is reduced in the PS mechanism (because $k$ is a threshold bidder), then we must have $b_{k}=m_{k}=p_{1}$, and so $b_{k} \prec b_{i}$ for all $i \in P, i \neq k$. Thus in this case bidder $k$ is the last bidder in $P$ to be considered by GFP. From here it is straightforward to show that GFP will assign the first $\ell^{*}$ slots to the bidders in $P$ (almost) exactly like the PS mechanism does, with at least $c_{i}-\epsilon^{\prime}$ clicks to each $i \in P$; the mechanism will have $\epsilon^{\prime}$ clicks left over, which will be assigned to bidders not in $P$. Applying this same argument to subsequent price blocks, we conclude that GFP will assign $c_{i}^{\prime}=c_{i} \pm \epsilon^{\prime}$ clicks to all bidders $i$.

To show this is an equilibrium, consider a bidder Alice (call her "bidder $a$ ") that was assigned to price block $j^{*}$ and received $c_{a}^{\prime}=c_{a} \pm \epsilon^{\prime}$ clicks. If Alice spent within $\epsilon^{\prime}$ of her entire budget, it means she would not want to raise her bid, since she could not possibly receive more than $\epsilon^{\prime}$ additional clicks. If she did not spend her budget, then from the observations above we know that she is bidding her true max-cpc $m_{a}$, and therefore also does not want to raise her bid.

It remains to show that Alice does not want to lower her bid. Let $\ell_{j}$ denote the last slot in price block $j$. Let $P_{j}$ denote the set of bidders in price block $j$. Alice's current bid $b_{a}$ is at least $p_{j}$, and if she keeps her bid above $p_{j}$ her clicks will remain $c_{a} \pm \epsilon$ from the arguments above. Let $S=\cup_{j \leq j^{*}} P_{j}$. If Alice lowers her bid to $b_{a}^{\prime}<p_{j}$, then all bidders $i \in S$ besides Alice will have $b_{i} \succ b_{a}^{\prime}$. Thus when Alice is considered by the greedy algorithm, her clicks will be constrained by the commitments to these bidders. Furthermore each of these bidders will still receive 
at least $c_{i}^{\prime}$ clicks. For all price blocks $j$, we have $\sum_{i \in P_{j}} c_{i}^{\prime} \geq \sum_{i=\ell_{j-1}+1}^{\ell_{j}} D_{i}-\epsilon^{\prime}$. Thus $\sum_{i \in S, i \neq a} c_{i}^{\prime} \geq\left(\sum_{i=1}^{\ell_{j *}} D_{i}\right)-\epsilon^{\prime}-c_{a}^{\prime}$. Since $S$ has size $\ell_{j^{*}}$, this implies that the constraint (3) restricts Alice's clicks to at most $c_{a}^{\prime}+\epsilon^{\prime}$. 